\title{
Coupled Anti Fuzzy Subrings Using $t$-Conorms
}

\author{
Clement Boateng Ampadu
}

31 Carrolton Road, Boston, MA 02132-6303, USA

e-mail: drampadu@hotmail.com

\begin{abstract}
The aim of this paper is to introduce the concept of coupled anti fuzzy subrings by using $t$-conorm $C$. By using $t$-conorm $C$; we consider the relationship between coupled subrings and coupled anti fuzzy subrings and we prove that the intersection of two coupled anti fuzzy subrings are also coupled anti fuzzy subring. Also we obtain some results for coupled anti fuzzy subrings under the ring homomorphisms. Finally, we show that the quotient of coupled anti fuzzy subring is also a coupled anti fuzzy subring with respect to $t$-conorm $C$. Our work is inspired by 1 .
\end{abstract}

\section{Some Old and New Notions and Notations}

Definition 1.1. Let $X$ be a set. A fuzzy subset of $X \times X$ will be a function from $X \times X$ into [0,1]. The set of all fuzzy subsets of $X \times X$ will be called the $[0,1]$-power set of $X \times X$, and will be denoted by $[0,1]^{X \times X}$.

Definition 1.2. 2] A $t$-conorm $C$ is a function $C:[0,1] \times[0,1] \mapsto[0,1]$ having the following four properties:

(a) $C(x, 0)=x$,

(b) $C(x, y) \leq C(x, z)$, if $y \leq z$

(c) $C(x, y)=C(y, x)$,

Received: December 1, 2020; Accepted: January 16, 2021

2010 Mathematics Subject Classification: 13Axx, 03B45, 03E72, $20 \mathrm{~K} 25$.

Keywords and phrases: ring theory, norms, fuzzy set theory. 
(d) $C(x, C(y, z))=C(C(x, y), z)$

for all $x, y, z \in[0,1]$.

Example 1.3. The basic $t$-conorms are standard union, $C_{m}(x, y)=\max \{x, y\}$. Bounded sum, $C_{b}(x, y)=\min \{1, x+y\}$, and algebraic sum, $C_{p}(x, y)=x+y-x y$ for all $x, y \in[0,1]$.

Definition 1.4. The $t$-conorm $C$ is called idempotent if for all $x \in[0,1], C(x, x)=$ $x$.

Lemma 1.5. [3] Let $C$ be a t-conorm. Then

$$
C(C(x, y), C(w, z))=C(C(x, w), C(y, z))
$$

for all $x, y, z, w \in[0,1]$.

Definition 1.6. (Compare with [4]) Let $f$ be a mapping from ring $R$ into $S$, $\mu \in[0,1]^{R \times R}, \beta \in[0,1]^{S \times S}$. Define $f(\mu) \in[0,1]^{S \times S}$ and $f^{-1}(\beta) \in[0,1]^{R \times R}$ such that for all $(y, v) \in S \times S$, if $f^{-1}(y), f^{-1}(v) \neq \emptyset$, then

$$
f(\mu)(y, v)=\sup \{\mu(x, m) \mid(x, m) \in R \times R, f(x)=y, f(m)=v\} .
$$

If $f^{-1}(y), f^{-1}(v)=\emptyset$, then

$$
f(\mu)(y, v)=0 .
$$

Theorem 1.7. (Compare with [5]) Let $R$ be a ring. A nonempty subset $S$ of $R \times R$ is a coupled subring of $R \times R$ iff $(x-y, m-v) \in S$ and $(x y, m v) \in S$ for all $(x, m),(y, v) \in S$.

Definition 1.8. (Compare with [5]) Let $R$ be a ring, and $I$ be a nonempty subset of $R \times R$. We say $I$ is a left(right) ideal of $R \times R$ if for all $(x, m),(y, v) \in I$ and for all $r \in R,(x-y, m-v) \in I,(r x, r m) \in I[(x-y, m-v) \in I,(x r, m r) \in I]$.

Definition 1.9. Let $R$ be a ring, and $\mu$ be a fuzzy subset of $R \times R$. We say $\mu$ is an anti fuzzy subring of $R \times R$ under a $t$-conorm $C$ iff for all $(x, m),(y, v) \in R \times R$, we have the following 
(a) $\mu(x-y, m-v) \leq C[\mu(x, m), \mu(y, v)]$;

(b) $\mu(x y, m v) \leq C[\mu(x, m), \mu(y, v)]$.

Notation 1.10. By $A F(R \times R)$ we mean the set of all anti fuzzy subrings of $R \times R$ under $t$-conorm $C$.

Definition 1.11. Let $\mu_{1}, \mu_{2} \in A F(R \times R)$ and $(x, m) \in R \times R$. We define the following

(a) $\mu_{1} \subseteq \mu_{2}$ iff $\mu_{1}(x, m) \leq \mu_{2}(x, m)$;

(b) $\mu_{1}=\mu_{2}$ iff $\mu_{1}(x, m)=\mu_{2}(x, m)$;

(c) $\left(\mu_{1} \cap \mu_{2}\right)(x, m)=C\left[\mu_{1}(x, m), \mu_{2}(x, m)\right]$.

Remark 1.12. By $(c)$ and $(d)$ of Definition 1.2, we also have

(a) $\mu_{1} \cap \mu_{2}=\mu_{2} \cap \mu_{1}$;

(b) $\mu_{1} \cap \mu_{2} \cap \mu_{3}=\left(\mu_{1} \cap \mu_{2}\right) \cap \mu_{3}=\mu_{1} \cap\left(\mu_{2} \cap \mu_{3}\right)$.

Definition 1.13. Let $R$ be a ring, $I$ be an ideal of $R$ and $\mu_{I} \in A F(R \times R)$. If $x \neq i, m \neq j$, define $\mu: \frac{R \times R}{I} \mapsto[0,1]$ by $\mu(x+I, m+I)=C\left[\mu_{I}(x, m), \mu_{I}(i, j)\right]$, and if $x=i, m=j$, define $\mu: \frac{R \times R}{I} \mapsto[0,1]$ by $\mu(x+I, m+I)=1$, for all $(x, m) \in R \times R, i, j \in I$.

\section{Some Properties}

Proposition 2.1. Let $\mu_{1}, \mu_{2} \in A F(R \times R)$. Then $\mu_{1} \cap \mu_{2} \in A F(R \times R)$.

Proof. Let $(x, m),(y, v) \in R \times R$. Observe

$$
\begin{aligned}
\left(\mu_{1} \cap \mu_{2}\right)(x-y, m-v) & =C\left[\mu_{1}(x-y, m-v), \mu_{2}(x-y, m-v)\right] \\
& \leq C\left[C\left[\mu_{1}(x, m), \mu_{1}(y, v)\right], C\left[\mu_{2}(x, m), \mu_{2}(y, v)\right]\right] \\
& =C\left[C\left[\mu_{1}(x, m), \mu_{2}(x, m)\right], C\left[\mu_{1}(y, v), \mu_{1}(y, v)\right]\right] \\
& =C\left[\left(\mu_{1} \cap \mu_{2}\right)(x, m),\left(\mu_{1} \cap \mu_{2}\right)(y, v)\right] .
\end{aligned}
$$


Also

$$
\begin{aligned}
\left(\mu_{1} \cap \mu_{2}\right)(x y, m v) & =C\left[\mu_{1}(x y, m v), \mu_{2}(x y, m v)\right] \\
& \leq C\left[C\left[\mu_{1}(x, m), \mu_{1}(y, v)\right], C\left[\mu_{2}(x, m), \mu_{2}(y, v)\right]\right] \\
& =C\left[C\left[\mu_{1}(x, m), \mu_{2}(x, m)\right], C\left[\mu_{1}(y, v), \mu_{1}(y, v)\right]\right] \\
& =C\left[\left(\mu_{1} \cap \mu_{2}\right)(x, m),\left(\mu_{1} \cap \mu_{2}\right)(y, v)\right] .
\end{aligned}
$$

It follows that $\mu_{1} \cap \mu_{2} \in A F(R \times R)$.

Proposition 2.2. Let $\mu \in A F(R \times R)$ and $C$ be idempotent. Then for all $t \in[0,1]$, $R_{t}=\{(x, m) \in R \times R \mid \mu(x, m) \leq t\}$ is a coupled subring of $R \times R$.

Proof. Let $(x, m),(y, v) \in R_{t}$. Then

$$
\begin{aligned}
\mu(x-y, m-v) & \leq C[\mu(x, m), \mu(y, v)] \\
& \leq C(t, t) \\
& =t
\end{aligned}
$$

which implies $(x-y, m-v) \in R_{t}$. Also

$$
\begin{aligned}
\mu(x y, m v) & \leq C[\mu(x, m), \mu(y, v)] \\
& \leq C(t, t) \\
& =t
\end{aligned}
$$

which implies $(x y, m v) \in R_{t}$. Thus $R_{t}$ is a coupled subring of $R \times R$.

Proposition 2.3. Let $R$ be a ring, and $\mu$ be a fuzzy subset of $R \times R$, and $C$ be idempotent, such that for all $(x, m),(y, v) \in R \times R$, and $r \in R, \mu(x-y, m-v) \leq$ $C[\mu(x, m), \mu(y, v)]$ and $\mu(r x, r m) \leq \mu(x, m)$. Then

(a) $\mu(0,0) \leq \mu(x, m)$, for all $(x, m) \in R \times R$;

(b) $\mu(x, m)=\mu(-x,-m)$, for all $(x, m) \in R \times R$;

(c) For all $t \in[0,1], R_{t}=\{(x, m) \in R \times R \mid \mu(x, m) \leq t\}$ is a left ideal of $R \times R$; 
(d) $R_{0}=\{(x, m) \in R \times R \mid \mu(x, m)=\mu(0,0)\}$ is a left ideal of $R \times R$.

Proof. Let $(x, m) \in R \times R$.

(a): $\mu(0,0)=\mu(x-x, m-m) \leq C[\mu(x, m), \mu(x, m)]=\mu(x, m)$.

(b):

$$
\begin{aligned}
\mu(x, m) & =\mu(0-(-x), 0-(-m)) \\
& \leq C[\mu(0,0), \mu(-x,-m)] \\
& \leq C[\mu(-x,-m), \mu(-x,-m)] \\
& =\mu(-x,-m) \\
& =\mu(0-x, 0-m) \\
& \leq C[\mu(0,0), \mu(x, m)] \\
& \leq C[\mu(x, m), \mu(x, m)] \\
& =\mu(x, m) .
\end{aligned}
$$

So $\mu(x, m)=\mu(-x,-m)$.

(c): If $(x, m),(y, v) \in R_{t}, r \in R$, then, $\mu(x-y, m-v) \leq C[\mu(x, m),(y, v)] \leq$ $C[t, t]=t$ and $\mu(r x, r m) \leq C[\mu(r x, r m), \mu(r x, r m)] \leq C[\mu(x, m), \mu(x, m)] \leq$ $C[t, t]=t$. So, $(x-y, m-v),(r x, r m) \in R_{t}$, thus $R_{t}$ is a left ideal of $R \times R$.

(d): If $(x, m),(y, v) \in R_{0}, r \in R$, then, $\mu(x-y, m-v) \leq C[\mu(x, m),(y, v)] \leq$ $C[\mu(0,0), \mu(0,0)]=\mu(0,0)$ and $\mu(r x, r m) \leq C[\mu(r x, r m), \mu(r x, r m)] \leq$ $C[\mu(x, m), \mu(x, m)] \leq C[\mu(0,0), \mu(0,0)]=\mu(0,0)$. So, $(x-y, m-v),(r x, r m) \in$ $R_{0}$, thus $R_{0}$ is a left ideal of $R \times R$.

Proposition 2.4. Let $\mu \in A F(R \times R)$ and $C$ be idempotent. Then $\mu(x-y, m-$ $v)=\mu(-y,-v)$ iff $\mu(x, m)=\mu(0,0)$ for all $(x, m),(y, v) \in R \times R$.

Proof. Let $(x, m),(y, v) \in R \times R$ and $\mu(x-y, m-v)=\mu(-y,-v)$. By letting $y=v=0$, we get $\mu(x, m)=\mu(0,0)$. Conversely, suppose that $\mu(x, m)=\mu(0,0)$. 
Then from the previous Proposition $\mu(x, m) \leq \mu(x-y, m-v), \mu(-y,-v)$. Now

$$
\begin{aligned}
\mu(x-y, m-v) & \leq C[\mu(x, m), \mu(y, v)] \\
& \leq C[\mu(-y,-v), \mu(y, v)] \\
& =C[\mu(-y,-v), \mu(-y,-v)] \\
& =\mu(-y,-v) \\
& =\mu(x-y-x, m-v-m) \\
& \leq C[\mu(x-y, m-v), \mu(x, m)] \\
& \leq C[\mu(x-y, m-v), \mu(x-y, m-v)] \\
& =\mu(x-y, m-v) .
\end{aligned}
$$

So, $\mu(x-y, m-v)=\mu(-y,-v)$.

Proposition 2.5. Let $\mu \in A F(R \times R)$ and $S$ be a ring. Suppose that $f$ is onto homomorphism of $R$ into $S$. Then $f(\mu) \in A F(S \times S)$.

Proof. Let $s_{1}, s_{2}, s_{3}, s_{4} \in S$, then there exists $(x, m),(y, v) \in R \times R$ such that $s_{1}=f(x), s_{2}=f(y), s_{3}=f(m), s_{4}=f(v)$. Now

$$
\begin{aligned}
& f(\mu)\left(s_{1}-s_{2}, s_{3}-s_{4}\right) \\
= & \sup \left\{\mu(x-y, m-v) \mid s_{1}=f(x), s_{2}=f(y), s_{3}=f(m), s_{4}=f(v)\right\} \\
\leq & \sup \left\{C[\mu(x, m), \mu(y, v)] \mid s_{1}=f(x), s_{2}=f(y), s_{3}=f(m), s_{4}=f(v)\right\} \\
= & C\left[\sup \left\{\mu(x, m) \mid s_{1}=f(x), s_{3}=f(m)\right\}, \sup \left\{\mu(y, v) \mid s_{2}=f(y), s_{4}=f(v)\right\}\right] \\
= & C\left[f(\mu)\left(s_{1}, s_{3}\right), f(\mu)\left(s_{2}, s_{4}\right)\right] .
\end{aligned}
$$

Also

$$
\begin{aligned}
& f(\mu)\left(s_{1} s_{2}, s_{3} s_{4}\right) \\
= & \sup \left\{\mu(x y, m v) \mid s_{1}=f(x), s_{2}=f(y), s_{3}=f(m), s_{4}=f(v)\right\} \\
\leq & \sup \left\{C[\mu(x, m), \mu(y, v)] \mid s_{1}=f(x), s_{2}=f(y), s_{3}=f(m), s_{4}=f(v)\right\} \\
= & C\left[\sup \left\{\mu(x, m) \mid s_{1}=f(x), s_{3}=f(m)\right\}, \sup \left\{\mu(y, v) \mid s_{2}=f(y), s_{4}=f(v)\right\}\right] \\
= & C\left[f(\mu)\left(s_{1}, s_{3}\right), f(\mu)\left(s_{2}, s_{4}\right)\right] .
\end{aligned}
$$

Therefore, $f(\mu) \in A F(S \times S)$. 
Proposition 2.6. Let $S$ be a ring, and $\beta \in A F(S \times S)$. If $f$ is a homomorphism of $R$ into $S$, then $f^{-1}(\beta) \in A F(R \times R)$.

Proof. Let $(x, m),(y, v) \in R \times R$. Observe

$$
\begin{aligned}
f^{-1}(\beta)(x-y, m-v) & =\beta(f(x-y), f(m-v)) \\
& =\beta(f(x)-f(y), f(m)-f(v)) \\
& \leq C[\beta(f(x), f(m)), \beta(f(y), f(v))] \\
& =C\left[f^{-1}(\beta)(x, m), f^{-1}(\beta)(y, v)\right] .
\end{aligned}
$$

Also

$$
\begin{aligned}
f^{-1}(\beta)(x y, m v) & =\beta(f(x y), f(m v)) \\
& =\beta(f(x) f(y), f(m) f(v)) \\
& \leq C[\beta(f(x), f(m)), \beta(f(y), f(v))] \\
& =C\left[f^{-1}(\beta)(x, m), f^{-1}(\beta)(y, v)\right] .
\end{aligned}
$$

So, $f^{-1}(\beta) \in A F(R \times R)$.

Proposition 2.7. Let $R$ be a ring, and $I$ be an ideal of $R$. If $C$ is idempotent, then $\mu \in A F\left(\frac{R \times R}{I}\right)$.

Proof. Let $(x+I, m+I),(y+I, v+I) \in \frac{R \times R}{I}$, and $i, j \in I$, and $\mu_{I} \in A F(R \times R)$ such that $x \neq i \neq y, m \neq j \neq v$. Observe

$$
\begin{aligned}
& \mu[(x+I)-(y+I),(m+I)-(v+I)] \\
= & \mu[(x-y)+I,(m-v)+I] \\
= & C\left[\mu_{I}(x-y, m-v), \mu_{I}(i, j)\right] \\
\leq & C\left[C\left[\mu_{I}(x, m), \mu_{I}(y, v)\right], \mu_{I}(i, j)\right] \\
= & C\left[C\left[\mu_{I}(x, m), \mu_{I}(y, v)\right], C\left[\mu_{I}(i, j), \mu_{I}(i, j)\right]\right] \\
= & C\left[C\left[\mu_{I}(x, m), \mu_{I}(i, j)\right], C\left[\mu_{I}(y, v), \mu_{I}(i, j)\right]\right] \\
= & C[\mu(x+I, m+I), \mu(y+I, v+I)] .
\end{aligned}
$$


Also

$$
\begin{aligned}
\mu[(x+I)(y+I),(m+I)(v+I)] & =\mu[x y+I, m v+I] \\
& =C\left[\mu_{I}(x y, m v), \mu_{I}(i, j)\right] \\
& \leq C\left[C\left[\mu_{I}(x, m), \mu_{I}(y, v)\right], \mu_{I}(i, j)\right] \\
& =C\left[C\left[\mu_{I}(x, m), \mu_{I}(y, v)\right], C\left[\mu_{I}(i, j), \mu_{I}(i, j)\right]\right] \\
& =C\left[C\left[\mu_{I}(x, m), \mu_{I}(i, j)\right], C\left[\mu_{I}(y, v), \mu_{I}(i, j)\right]\right] \\
& =C[\mu(x+I, m+I), \mu(y+I, v+I)] .
\end{aligned}
$$

Finishing the proof.

\section{An Open Problem}

We begin by introducing the following

Definition 3.1. Let $R$ and $S$ be two rings such that $\mu \in A F(R \times R)$ and $\beta \in$ $A F(S \times S)$. The direct product of $\mu$ and $\beta$, denoted by $\mu \times \beta$, is the fuzzy subset of ring $R^{2} \times S^{2}$ such that for all $(x, m) \in R \times R$ and $(y, v) \in S \times S$, we have

$$
(\mu \times \beta)[(x, m),(y, v)]=C[\mu(x, m), \beta(y, v)] .
$$

Conjecture 3.2. If $\mu_{I} \in A F\left(R_{i} \times R_{i}\right)$ for $i=1,2$, then $\mu_{1} \times \mu_{2} \in A F\left(R_{1}^{2} \times R_{2}^{2}\right)$.

Conjecture 3.3. Let $\mu_{i} \in A F\left(R_{i} \times R_{i}\right)$ for $i=1,2, \cdots, n$. Then

$$
\mu_{1} \times \mu_{2} \times \cdots \times \mu_{n} \in A F\left(R_{1}^{2} \times R_{2}^{2} \times \cdots \times R_{n}^{2}\right) .
$$

\section{References}

[1] Rasul Rasuli, Some results of anti fuzzy subrings over $t$-conorms, MathLAB Journal 4 (2019), 25-32.

[2] J. J. Buckley and E. Eslami, An Introduction to Fuzzy Logic and Fuzzy Sets, Springer-Verlag, Berlin, Heidelberg GmbH, 2002.

https://doi.org/10.1007/978-3-7908-1799-7 
[3] M. T. Abu Osman, On some products of fuzzy subgroups, Fuzzy Sets and Systems 24 (1987), 79-86. https://doi.org/10.1016/0165-0114(87)90115-1

[4] D. S. Malik and J. N. Mordeson, Fuzzy Commutative Algebra, World Science Publishing Co. Pte. Ltd., 1995.

[5] D. S. Malik, J. N. Mordeson and M. K. Sen, Fundamentals of Abstract Algebra, McGraw Hill, New York, 1997.

This is an open access article distributed under the terms of the Creative Commons Attribution License (http://creativecommons.org/licenses/by/4.0/), which permits unrestricted, use, distribution and reproduction in any medium, or format for any purpose, even commercially provided the work is properly cited. 